\title{
A Greedy Heuristic for Crossing-Angle Maximization ${ }^{\star}$
}

\author{
Almut Demel, Dominik Dürrschnabel, Tamara Mchedlidze, Marcel \\ Radermacher, and Lasse Wulf \\ Department of Computer Science, Karlsruhe Institute of Technology, Germany \\ mched@iti.uka.de, radermacher@kit.edu
}

\begin{abstract}
The crossing angle of a straight-line drawing $\Gamma$ of a graph $G=(V, E)$ is the smallest angle between two crossing edges in $\Gamma$. Deciding whether a graph $G$ has a straight-line drawing with a crossing angle of $90^{\circ}$ is $\mathcal{N} \mathcal{P}$-hard [1. We propose a simple heuristic to compute a drawing with a large crossing angle. The heuristic greedily selects the best position for a single vertex in a random set of points. The algorithm is accompanied by a speed-up technique to compute the crossing angle of a straight-line drawing. We show the effectiveness of the heuristic in an extensive empirical evaluation. Our heuristic was clearly the winning algorithm (CoffeeVM) in the Graph Drawing Challenge 2017 [6].
\end{abstract}

\section{Introduction}

The crossing angle $\operatorname{cr}-\alpha(\Gamma)$ of a straight-line drawing $\Gamma$ is defined to be the minimum over all angles created by two crossing edges in $\Gamma$. The 24th edition of the annual Graph Drawing Challenge, held during the Graph Drawing Symposium, posed the following problem: Given a graph $G$, compute a straight-line drawing $\Gamma$ on an integer grid that has a large crossing angle. In this paper we present a greedy heuristic that starts with a carefully chosen initial drawing and repeatedly moves a vertex $v$ to a random point $p$ if this increases the crossing angle of $\Gamma$. This heuristic was the winning algorithm of the GD Challenge '17 [6].

Related Works A drawing of a graph is called $R A C$ if its minimum crossing angle is $90^{\circ}$. Deciding whether a graph has a straight-line RAC drawing is an $\mathcal{N} \mathcal{P}$-hard problem [1]. Giacomo et al.[13] proved that every straight-line drawing of a complete graph with at least 12 vertices has a crossing angle of $\Theta(\pi / n)$. Didimo et al. [7 have shown that every $n$-vertex graph that admits a straightline RAC drawing has at most $4 n-10$ edges. This bound is tight, since there is an infinite family of graphs with $4 n-10$ edges that have straight-line RAC drawings. Moreover they proved that every graph has a RAC drawing with three bends per edge. Arikishu et al. [3] showed that any $n$-vertex graph that admits a

\footnotetext{
* Work was partially supported by grant WA 654/21-1 of the German Research Foundation (DFG).
} 
(a)

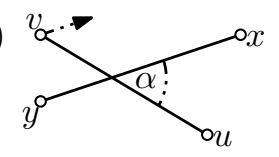

(b)

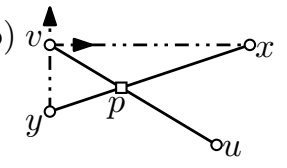

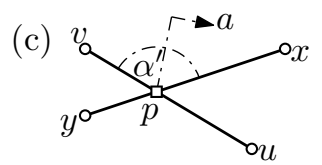

Fig. 1: Sketches of the force (a) $F_{\cos }(v)$, (b) $F_{\text {cage }}(v)$ and (c) $F_{\text {ang }}(v)$.

RAC drawing with one bend or two bends per edge has at most $6.5 n$ and $74.2 n$ edges, respectively. For an overview over further results on RAC drawings we refer to [8]. Dujmović et al. [9] introduced the concept of $\alpha \mathrm{AC}$ graphs. A graph is $\alpha A C$ if it admits a drawing with crossing angle of at least $\alpha$. For $\alpha>\pi / 3, \alpha \mathrm{AC}$ graphs are quasiplanar graphs, i.e., graphs that admit a drawing without three mutually crossing edges, and thus have at most $6.5 n-20$ edges. Moreover, every $n$-vertex $\alpha \mathrm{AC}$ graph with $\alpha \in(0, \pi / 2)$ has at most $(\pi / \alpha)(3 n-6)$ edges. Besides the theoretical work on this topic, there are a few force-directed approaches that optimize the crossing angle in drawings of arbitrary graphs [14/2], see Sec. 2.1.

Contribution We introduce a heuristic to increase the crossing angle in a given straight-line drawing $\Gamma$ (Sec. 3). The heuristic is accompanied by a speed-up technique to compute the pair of crossing edges in $\Gamma$ that create the smallest crossing angle. In Sec. 4 we give an extensive evaluation of our heuristic. The evaluation is driven by three main research questions: i) What is a good parametrization of our heuristic? ii) Does our heuristic improve the crossing angle of a given initial drawing? iii) What is a good choice for an initial drawing?

\section{Preliminaries}

Let $\Gamma$ be a straight-line drawing of a graph $G=(V, E)$. Denote by $n$ and $m$ the number of vertices and edges of $G$, respectively. Let $e$ and $e^{\prime}$ be two distinct edges of $G$. If $e$ and $e^{\prime}$ have an interior intersection in $\Gamma$, the function $\operatorname{cr}-\alpha\left(\Gamma, e, e^{\prime}\right)$ denotes the smallest angle formed by $e$ and $e^{\prime}$ in $\Gamma$. In case that $e$ and $e^{\prime}$ do not intersect, we define $\operatorname{cr}-\alpha\left(\Gamma, e, e^{\prime}\right)$ to be $\pi / 2$. The local crossing angle of a vertex $v$ is defined as the minimum angle of the edges incident to $v$, i.e., $\operatorname{cr}-\alpha(\Gamma, v)=$ $\min _{e, u v \in E, e \neq u v} \operatorname{cr}-\alpha(\Gamma, e, u v)$. The crossing angle of a drawing $\Gamma$ is defined as $\operatorname{cr}-\alpha(\Gamma)=\min _{e, e^{\prime} \in E, e \neq e^{\prime}} \operatorname{cr}-\alpha\left(\Gamma, e, e^{\prime}\right)$. Let $\Delta x$ and $\Delta y$ be the difference of the $\mathrm{x}$-coordinates and the y-coordinates of the endpoints of $e$ in a drawing $\Gamma$. The slope of $e$ is the angle between $e$ and the $x$-axis, i.e. $\operatorname{slope}(\Gamma, e)=\arctan (\Delta y / \Delta x)$ if $\Delta x \neq 0$ and slope $(\Gamma, e)=-\pi / 2$ otherwise.

\subsection{Force-directed Approaches}

In general, force-directed algorithms [11|10] compute for each vertex $v$ of a graph $G=(V, E)$ a force $F_{v}$. A new drawing $\Gamma^{\prime}$ is obtained from a drawing $\Gamma$ by displacing every vertex $v$ according to the force $F_{v}$. Classically, the force $F_{v}$ is 


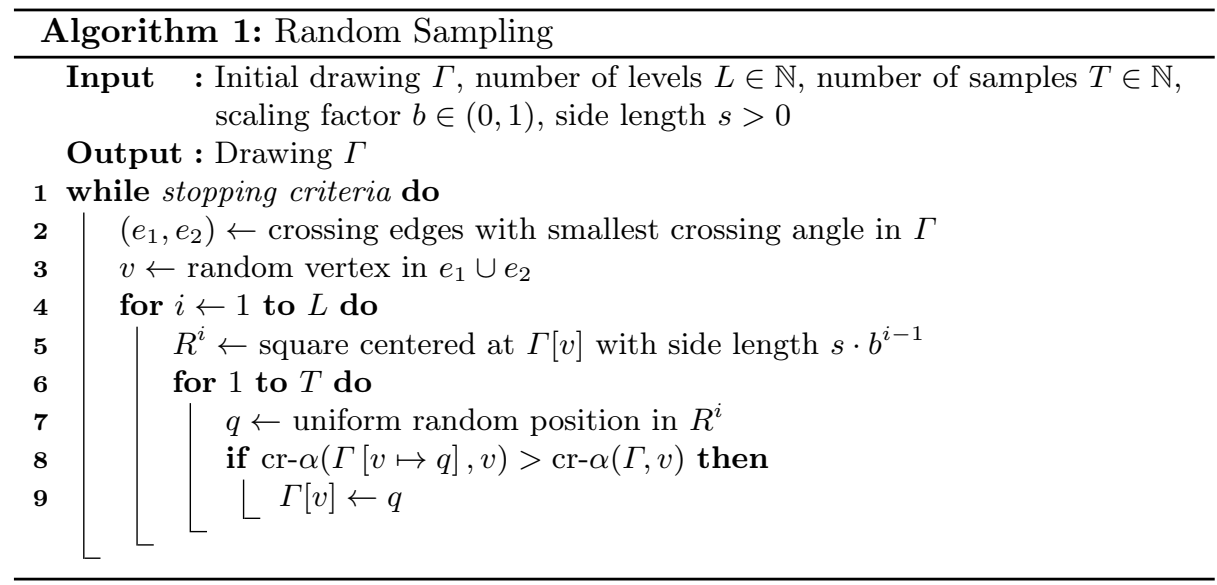

a linear combination of repelling and attracting forces, i.e., all pairs of vertices repel each other, and incident vertices attract each other. It is easy to integrate new forces into this generic system, e.g., in order to increase the crossing angle. For this purpose, Huang et al. [14 introduced the cosine force $F_{\mathrm{cos}}$. The forcedirected approach considered by Argyriou et al. [2] uses two forces, $F_{\text {cage }}$ and $F_{\text {ang }}$, to increase the crossing angle. In the following we will describe each force.

Let $\overrightarrow{x y}$ denote the unit length vector from $x$ to $y$. Let $u v, x y$ be two crossing edges in $\Gamma$ and let $\alpha$ be the angle as depicted in Fig. 1(a) and let $p$ denote the intersection point of $u v$ and $x y$, see Fig 1 . The cosine force for $v$ is defined as $F_{\cos }(v)=k_{\cos } \cdot \cos \alpha \cdot \overrightarrow{y x}$, where $k_{\cos }$ is a positive constant.

The force $F_{\text {cage }}(v)$ is a compound of two forces $F_{\text {cage }}(v, x)$ and $F_{\text {cage }}(v, y)$. Let $l_{a b}$ denote the distance between two points $a$ and $b$. Let $l_{v x}^{\star}$ be the length of the edge $v x$ in a triangle $v x p$ with side length $l_{v p}$ and $l_{x p}$, and a right angle at the point $p$. Then, $F_{\text {cage }}(v, x)=k_{\text {cage }} \cdot \log \left(l_{v x} / l_{v x}^{\star}\right) \overrightarrow{v x}$, where $k_{\text {cage }}$ is positive constant. The force $F_{\text {cage }}(v, y)$ is defined symmetrically.

Again the force $F_{\text {ang }}(v)$ is a compound of the forces $F_{\text {ang }}(v, x)$ and $F_{\text {ang }}(v, y)$. Consider the unit vector $a$ that is perpendicular to the bisector of $\overrightarrow{u v}$ and $\overrightarrow{y x}$, refer Fig. 1. Further, let $\alpha^{\prime}$ be the angle between the $\overrightarrow{u v}$ and $\vec{y} \vec{x}$. Then the force $F_{\text {ang }}(v, x)$ is defined as $k_{\text {ang }} \cdot \operatorname{sign}\left(\alpha^{\prime}-\pi / 2\right) \cdot\left|\pi / 2-\alpha^{\prime}\right| / \alpha^{\prime} \cdot a$ where $k_{\text {ang }}$ is a positive constant. The force $F_{\text {ang }}(v, y)$ is defined correspondingly.

\section{Multilevel Random Sampling}

Our algorithm starts with a drawing $\Gamma$ of a graph $G$ and iteratively improves the crossing angle of $\Gamma$ by moving a vertex to a better position, i.e., we locally optimize the crossing angle of the drawing; for pseudocode refer to Alg. 1. For this purpose we greedily select a vertex $v$ with a minimal crossing angle $\operatorname{cr}-\alpha(\Gamma, v)$. More precisely, let $e$ and $e^{\prime}$ be two edges with a minimal crossing angle in $\Gamma$. We set $v$ randomly to be an endpoint of $e$ and $e^{\prime}$. We iteratively improve the 
crossing angle of $v$ by sampling a set $S$ of $T$ points within a square $R$ and by moving $v$ to the position $p \in S$ that induces a maximal local crossing angle. We repeat this process $L \in \mathbb{N}^{+}$times and decrease the size of $R$ in each iteration.

More formally, denote by $\Gamma[v \mapsto p]$ the drawing obtained from $\Gamma$ by moving $v$ to the point $p=\left(p_{x}, p_{y}\right) \in \mathbb{R}^{2}$. Let $R^{i}(p)=\left[p_{x}-s \cdot b^{i} / 2, p_{y}-s \cdot b^{i} / 2\right] \times\left[p_{x}+\right.$ $\left.s / 2, p_{y}+s \cdot b^{i} / 2\right] \subset \mathbb{R}^{2}$ be a square centered at the point $p$ with a scaling factor $b \in(0,1)$ and initial side length $s>0$. Let $p^{0}$ be the position of $v$ in $\Gamma$ and let $S^{0} \subset R^{0}\left(p^{0}\right)$ be a set of $T$ points in $R^{0}\left(p^{0}\right)$ chosen uniformly at random. Let $p^{i}$ be a point in $S^{i-1} \cup\left\{p^{i-1}\right\}$ that maximizes $\operatorname{cr}-\alpha\left(\Gamma\left[v \mapsto p^{i}\right], v\right)$. We obtain a new sample $S^{i}$ by randomly selecting $T$ points within the square $R^{i}\left(p^{i}\right)$. Since $\operatorname{cr}-\alpha\left(\Gamma\left[v \mapsto p^{i}\right], v\right)=\max _{u v \in E, e \in E \backslash\{u v\}} \operatorname{cr}-\alpha\left(\Gamma\left[v \mapsto p^{i}\right], u v, e\right)$, the function can be evaluated in $O(\operatorname{deg}(v)|E|)$ time.

\subsection{Fast Minimum Angle Computation}

The running time of the random sampling approach relies on computing in each iteration a pair of edges creating the minimum crossing angle $\mathrm{cr}-\alpha(\Gamma)$. More formally, we are looking for a pair of distinct edges $e, f \in E$ that have a minimal crossing angle in a straight-line drawing $\Gamma$, i.e., $\operatorname{cr}-\alpha(\Gamma, e, f)=\operatorname{cr}-\alpha(\Gamma)$. The well known sweep-line algorithm [4] requires $O((n+k) \log (n+k))$ time to report all $k$ intersecting edges in $\Gamma$. In general the number of intersecting edges can be $\Omega\left(m^{2}\right)$, but we are only interested in a single pair that forms the minimal crossing angle. Therefore, we propose an algorithm, which uses the slopes of the edges in $\Gamma$ to rule out pairs of edges, which cannot form the minimum angle.

Assume that we already found two intersecting edges forming a small angle of size $\delta>0$. We set $t:=\lfloor\pi / \delta\rfloor$ and distribute the edges into $t$ buckets $B_{0}, \ldots, B_{t-1}$ such that bucket $B_{i}$ contains exactly the edges $e$ with $i \pi / t \leq \operatorname{slope}(\Gamma, e)+\pi / 2<$ $(i+1) \pi / t$. Then each bucket covers an interval of size $\pi /\lfloor\pi / \delta\rfloor \geq \delta$. Thus, if there exist edges $e, f$ with $\mathrm{cr}-\alpha(\Gamma, e, f)<\delta$, they belong to the same or to the adjacent buckets (modulo $t$ ). Overall, we consider all pairs of edges in $B_{i} \cup B_{i+1}(\bmod t)$, $i=1, \ldots t$, and find the pair forming the smallest crossing angle. To find this pair we could apply a sweep-line algorithm to the set $B_{i} \cup B_{i+1}$. In general this set can contain $\Omega(m)$ edges. Thus, in worst case we would not gain a speed up in comparison to a sweep-line algorithm applied to $\Gamma$. On the other hand, in practice we expect the number of edges in a bucket to be small. If we assume this number to be a constant, the overall running time of the exhaustive check is linear in $m$ and does not depend on the number of crossings.

Implementation Details. In the case that the slopes in $\Gamma$ are uniformly distributed, we expect the number of edges in a bucket to decrease with an decreasing estimate $\delta$. We set the value $\delta$ to be the minimal crossing angle of the $r$ longest edges in $\Gamma$. In our implementation we set $r$ to be 50 if the graph contains at most 5000 edges, otherwise it is 300 . 

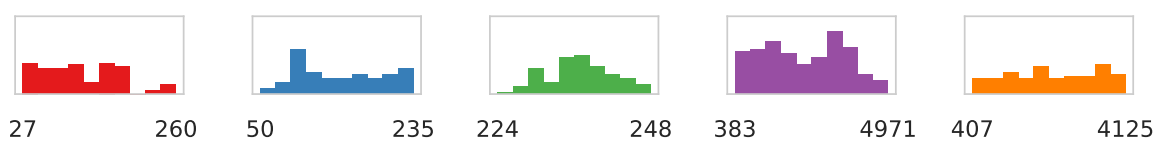

(a) NorTH

(b) Rome

(c) Community

(d) 1-Planar

(e) TRI. $+\mathrm{X}$

Fig. 2: The distribution of the sum of number of vertices and edges per graph class. The plot is scaled such that a bar of full height would contain 40 graphs.

\section{Experimental Evaluation}

The RANDOM SAMPLING heuristic has several parameters which allow for many different configurations. In Sec. 4.4, we investigate the influence of the configuration on the crossing angle of the drawing computed by the random sampling approach. We investigate the question of whether the RANDOM SAMPLING approach improves the crossing angle of a given drawing. Our evaluation in Sec. 4.5 answers the question affirmatively. Moreover, we expect that the crossing angle of the drawing computed by the random sampling approach depends on the choice of the initial drawing. We show that this is indeed the case (Sec. 4.6). We close the evaluation with a short running time analysis in Sec. 4.7. Our evaluation is based on a selection of artificial and real world graphs (Sec. 4.1), several choices of the initial drawing, see Sec. 4.2, and a specific way to compare two drawing algorithms (Sec 4.3).

Setup. All experiments were conducted on a single core of an AMD Opteron Processor 6172 clocked at $2.1 \mathrm{GHz}$. The server is equipped with 256GB RAM. All algorithms were compiled with $\mathrm{g}++-4.8 .5$ with optimization mode -03 .

\subsection{Benchmark Graphs}

We evaluate the heuristic on the following graph classes, either purely synthetic or with a structure resembling real-world data. Fig. 2 shows the size distribution of these graphs. The color of each class is used consistently throughout the paper.

Real World. The classes Rome and North (AT\&T) ${ }^{1}$ are the non-planar subsets of the corresponding well known benchmark sets, respectively. From each graph class we picked 100 graphs uniformly at random. The CommuniTy graphs are generated with the LFR-GEnERATOR [17] implemented in NeTwORKIT [19]. These graphs resemble social networks with a community structure.

Artificial. For each artificial graph we picked the number $n$ of vertices uniformly at random between 100 and 1000. The TRIANGUlation $+\mathrm{X}$ class contains randomly generated $n$-vertex triangulations with an additional set of $x$ edges. The number $x$ is picked uniformly at random between $0.1 n$ and $0.15 n$. The endpoints of the additional edge are picked uniformly at random, as well.

\footnotetext{
${ }^{1}$ http://graphdrawing.org/data.html
} 


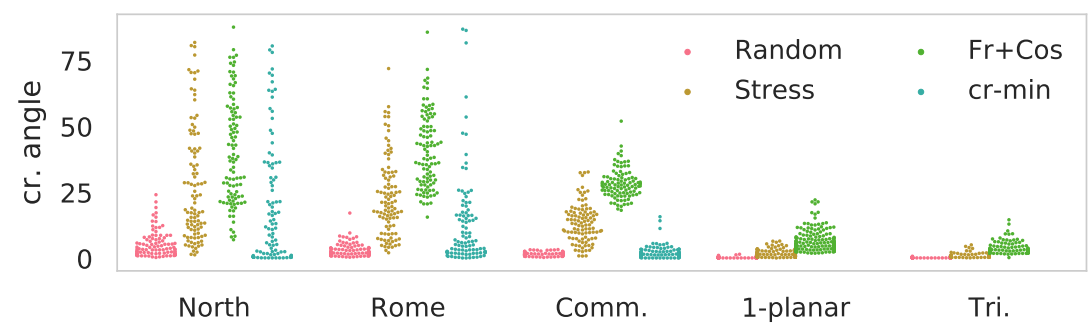

Fig. 3: Crossing angles of the initial drawings.

The class 1-PlanAR consists of graphs that admit drawings where every edge has at most one crossing. We used a geometric and topological procedure to generate these graphs. For the former consider a random point set $P$ of $n$ points. Let $e_{1}, \ldots e_{k}$ be a random permutation of all pairs of points in $P$. Let $G_{0}=(P, \emptyset)$. If the drawing $G_{i-1}+e_{i}$ induced by $P$ is simple and 1-planar, we define $G_{i}$ to be this graph, otherwise we set $G_{i}=G_{i-1}$. We construct the topological 1-PlanaR graphs based on a random planar triangulation $G$ generated with OGDF [5]. Let $v$ be a random vertex of $G$ and let $v, x, u, y$ be an arbitrary 4-cycle. We add $u v$ to $G$ if $G+u v$ is 1-planar. The process is repeated $x$ times, for a random number $x \in[0.3 n, 0.4 n]$. In contrast to the experimental work on crossing minimization in book embeddings [16], we did not observe that our heuristic performs differently on the topological and geometric 1-Planar graphs, see Appendix A. Hence, we merge the two classes into a single class. Thus, in total the 1-PlanAR graphs contain 200 graphs compared to 100 in the other graph classes.

\subsection{Initial Drawings}

In our evaluation we consider four initial drawings of each benchmark graph; refer to Tab. 1a. A random point set $P$ of size $n$ induces a RANDOM drawing of an $n$-vertex graph. The FR + Cos drawings are generated by applying our implementation of the force-directed method of Fruchtermann and Reingold [11] to the RANDOM drawings with the additional $F_{\text {cos }}$ force (Sec. 2.1). We applied the stress majorization [5]12] implementation of the OPEN GRAPH DraWING Framework (OGDF) to RANDOM in order to obtain the STRESS drawings. The CR-SMALL drawings are computed with the heuristic introduced by Radermacher et al. [18 in order to decrease the number of crossings in straight-line drawings. They showed that the heuristic computes drawings with significantly less crossings than drawings computed by stress majorization. Unfortunately, within a feasible amount of time we were not able to compute CR-SMALL drawing for the classes 1-Planar and TRIANGULATION $+\mathrm{X}$.

A point in Fig. 3 corresponds to the crossing angle of an initial drawing. The plot is categorized by graph class. The RANDOM drawings have the smallest crossing angles. The STRESS drawings have a larger crossing angle than CRSMALL and overall, Fr + Cos drawings tend to have the largest crossing angle. 
Identifier Algorithm

\begin{tabular}{ll}
\hline RANDOM & uni. rand. vertex placement \\
FR+Cos & FR + Cosine Forces (Sec. 2.1 \\
STRESS & Stress Majorization [12] \\
CR-SMALL & Crossing Minimization [18
\end{tabular}

(a)

\begin{tabular}{lcc} 
& Levels & Sample Size \\
& $L$ & $T$ \\
\hline Sloppy & 3 & 50 \\
MEDIUM & 4 & 175 \\
PreCISE & 5 & 400
\end{tabular}

(b)

Table 1: (a) Initial drawings with their identifiers used throughout the paper. (b) Configurations of the RANDOM SAmPLING approach. The scaling factor $b$ is 0.2 and the initial side length $s$ is $10^{5}$.

We point out that in contrast to the evaluation of Argyriou et al. 2], our implementation of the force-directed method with $F_{\text {cage }}$ and $F_{\text {ang }}$ produces drawings with smaller crossing angles than with $F_{\text {cos }}$. Thus, we do not consider these drawings in our evaluation. For the further details refer to Appendix B.

\subsection{Differences between Paired Drawings}

In order to compare the performance of two algorithms on multiple graphs and to investigate by how much one of the algorithms outperforms the other, we employ the following machinery. We denote by $\Gamma\{G\}$ the set of all drawings of $G$. Let $\mathcal{G}=\left\{G_{1}, G_{2}, \ldots, G_{k}\right\}$ be a family of (non-planar) graphs. We refer to a set $\Lambda=\left\{\Gamma_{1}, \ldots, \Gamma_{k}\right\}$ as a family of drawings of $\mathcal{G}$ where $\Gamma_{i} \in \Gamma\left\{G_{i}\right\}$. Let $\Lambda^{1}$ and $\Lambda^{2}$ be two families of drawings of $\mathcal{G}$. Let $\mathcal{F}$ be a subset of $\mathcal{G}$. We say $\Lambda^{1}$ outperforms $\Lambda^{2}$ on $\mathcal{F}$ if and only if for all $G_{i} \in \mathcal{F}$ the inequality $\operatorname{cr}-\alpha\left(\Gamma_{i}^{1}\right)>\operatorname{cr}-\alpha\left(\Gamma_{i}^{2}\right)$ holds. If $\Lambda^{1}$ outperforms $\Lambda^{2}$ on $\mathcal{F}$ then $\Lambda^{1}$ has an advantage of $\Delta>0$ on $\mathcal{F}$ if for all $G_{i} \in \mathcal{F}$ the inequality $\operatorname{cr}-\alpha\left(\Gamma_{i}^{1}\right)>\operatorname{cr}-\alpha\left(\Gamma_{i}^{2}\right)+\Delta$ holds. For a finite set $\mathcal{G}$, we say $\mathcal{F}$ has relative size at least $p \in[0,1]$ if $|\mathcal{F}| \geq p \cdot|\mathcal{G}|$.

In order to compare two families of drawings we plot the advantage as a function of $p$; refer to Fig. 5 For each value $p$ the plot contains 5 five bars, each corresponding to a graph class. The height of the bars correspond to advantages $\Delta$ for a set of relative size $p$. A caption of a figure in the form of $A$ vs $B$ indicates that if $\Delta$ is positive, $B$ has advantage $\Delta$ over A. Correspondingly, if $\Delta$ is negative, $A$ has an advantage of $-\Delta$ over $B$. Thus, Fig. 5 shows that for $p=0.1$, for each graph class there is a subset $\mathcal{F}$ of relative size 0.1 , i.e., $\mathcal{F}$ contains at least 10 graphs, such that the set SLOPPY has an advantage of $\Delta$ over PRECISE on $\mathcal{F}$. In greater detail, SLOPPy has an advantage of $7.9^{\circ}$ over Precise on the NoRTH graphs, $12.9^{\circ}$ on the Rome graphs, $11.5^{\circ}$ on the COMMUnity graphs, $1.2^{\circ}$ on the 1-Planar graphs and $1.2^{\circ}$ on the Triangulation $+\mathrm{X}$ graphs. On the other side, PRECISE has an advantage of $12.9^{\circ}$ over SLOPPY on at least 10 NoRTh graphs, $15.7^{\circ}$ on the Rome graphs, $13.8^{\circ}$ on the Community graphs, $1.1^{\circ}$ on the 1 -Planar graphs and $0.4^{\circ}$ on the Triangulation $+\mathrm{X}$ graphs. Note that only for $p<0.5$ there can be two disjoint subsets $\mathcal{F}_{1}, \mathcal{F}_{2}$ of a graph class 


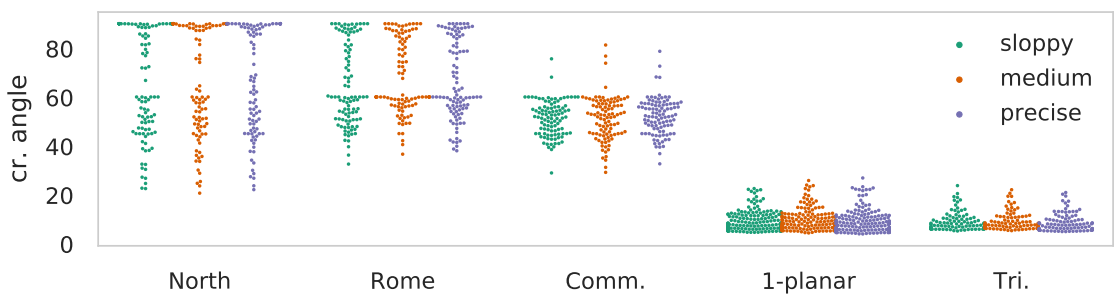

Fig. 4: Performance of different configurations

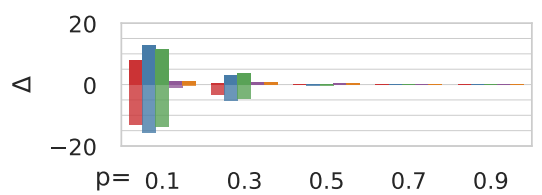

(a) Precise vs Sloppy

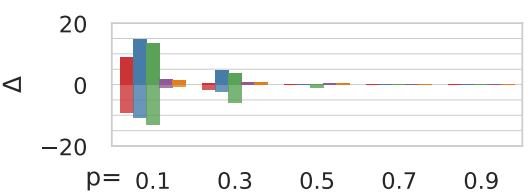

(b) Precise vs Medium

Fig. 5: Comparison of the Sloppy configuration to the Medium and Precise configuration. The colors indicate the graph as indicated by Fig. 2 .

of relative size $p$ such that Precise has an advantage over Sloppy on $\mathcal{F}_{1}$ and SlOPPY has an advantage over PRECISE on $\mathcal{F}_{2}$.

\subsection{Parametrization of the Random Sampling Approach}

The RANDOM SAmPling approach introduced in Section 3 has four different parameters, the number of levels $L$, the size of the sample $T$, the initial side length $s$ and the scaling factor $b$, that allows for many different configurations. With an increasing number $T$ of samples, we expect to obtain a larger crossing angle in each iteration to the cost of an increasing running time. If we allow each configuration the same running time, it is unclear whether it is beneficial to increase the number of iterations or to increase the number of samples $(T)$ and levels $(L)$ per iteration. This motivates the following question: does the crossing angle of a drawing of an $n$-vertex graph computed by the random sampling approach within a given time limit $t_{n}$ increase with an increasing number of samples and levels? We choose to set the time limit $t_{n}$ to $n$ seconds. This allows for at least $1.6 \cdot n$ iterations on our benchmark instances. For further details see Appendix D Since the parametrization space is infeasibly large, we evaluate three exemplary configurations, SLoppy, Medium and Precise; see Tab. 1b.

The plot in Fig. 4 does not indicate that the distributions of the crossing angle differ across different configurations significantly ; further characteristics are listed in Tab. 2 in the appendix. With the plot in Fig. 5 we can confirm this observation. For each configuration there is only a small subset of each class such that the configuration has an advantage over the other configurations. For example, for the Rome graphs there exist at least 10 graphs such that SLOPPY 


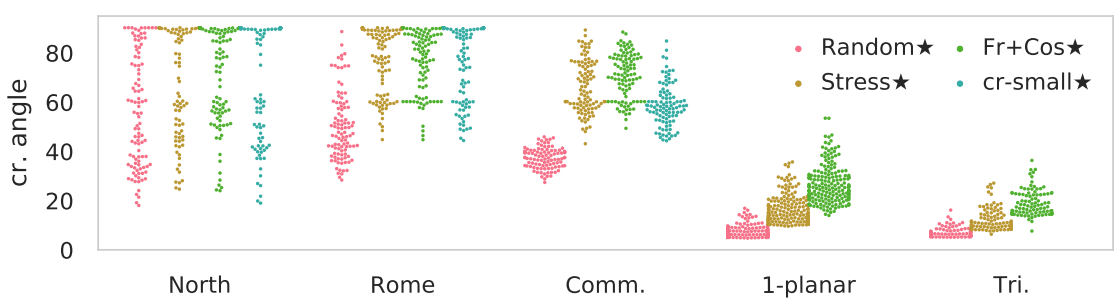

Fig. 6: Crossing angles of the initial drawings after optimization with the RANDOM SAMPLING approach.

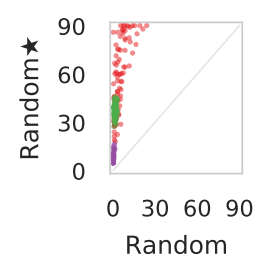

(a) RANDOM

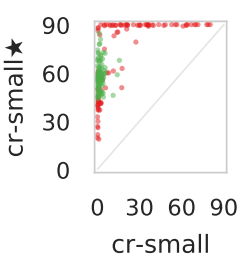

(b) CR-SMall

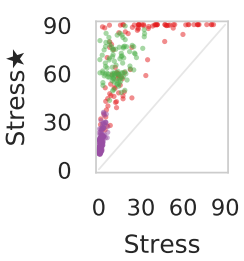

(c) Stress

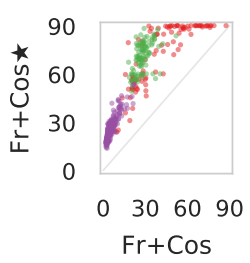

(d) $\mathrm{Fr}+\mathrm{Cos}$

Fig. 7: Initial crossing angle vs the final crossing angle. The plots show the crossing angles of the classes NorTh, COMMUNiTy and 1-Planar.

has an advantage of $10^{\circ}$ over PRECISE. On the other hand, there are at least 10 different graphs such PRECISE has also an advantage of $10^{\circ}$ over SlOPPY. For $p \geq 0.5$ no configuration has an advantage over the other, or it negligibly small. Thus, we conclude that given a common time limit, increasing the levels and the sample size does not necessarily increase the crossing angle.

\subsection{Improvement of the Crossing Angles}

In this section we investigate whether the RANDOM SAMPLING approach is able to improve the crossing angle of a given drawing within $2 n$ iterations. Given the same number of iterations, it is most-likely that we obtain a larger crossing angle of a drawing if we increase the number of samples. Thus, we use the PRECISE configuration for the evaluation of the above question. We refer to the drawings after the application of the RANDOM SAMPLING approach as RAN$\mathrm{DOM}^{\star}, \mathrm{Fr}+\mathrm{COS}^{\star}, \mathrm{STRESS}^{\star}$ and CR-SMALL${ }^{\star}$, respectively. For characteristics of the crossing angles refer to Tab. 3 in the appendix.

The plots in Fig. 3 and Fig. 6 indicate that the RANDOM SAmPLing approach indeed improves the crossing angle of the initial drawings. Fig. 7 shows the relationship between the crossing angle of the initial drawing and the final drawing. For the purpose of clarity, the plot only shows drawings of the classes NORTH, Community and 1-Planar; for the remaining classes refer to Appendix E. The plots shows that the RANDOM SAMPLING approach considerably improves 


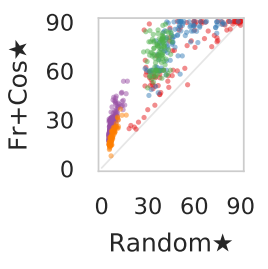

(a) RANDOM

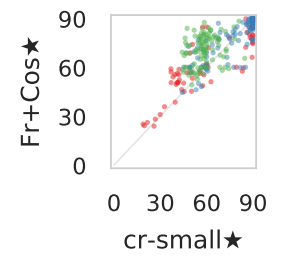

(b) Cr-Small

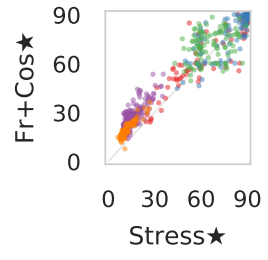

(c) Stress

Fig. 8: Comparison of the initial layout.

the crossing angle of the initial drawing. In case of the NORTH graphs there are a few graphs that have an improvement of at least $70^{\circ}$. There are at least 10 drawings in RANDOM whose crossing angle is improved by at least $75^{\circ}$; refer to Fig. 16 in Appendix E. For all real world graph classes and all initial layouts there are 70 graphs in each class, such that the final drawing has an advantage of over $25^{\circ}$.

For Triangulation $+\mathrm{X}, \mathrm{Fr}+\mathrm{Cos}^{\star}$ has an advantage of at least $11^{\circ}$ over $\mathrm{Fr}+\mathrm{COS}$ on at least 90 Triangulation $+\mathrm{X}$. For the remaining initial layouts the corresponding advantage is at most $7.6^{\circ}$. Considering the 1-PlanaR graphs the corresponding advantages are $14^{\circ}$ and $9.7^{\circ}$. This indicates that within $2 n$ iterations a large initial crossing angle helps to further improve the crossing angle of 1-Planar and Triangulation $+\mathrm{X}$ graphs. Overall we observe that the 1-Planar and Triangulation $+\mathrm{X}$ classes are rather difficult to optimize. This can either be a limitation of our heuristic or the crossing angle of these graphs are indeed small. Unfortunately, we are not aware of meaningful upper and lower bounds on the crossing angle of straight-line drawing of these graphs. Nevertheless, we can conclude that our heuristic indeed improves the initial crossing angle. To which extend our heuristic is able to increase crossing angle of a drawing depends on the graph class and on the initial drawing itself.

\subsection{Effect of the Initial Drawing}

The RANDOM SAmpling approach iteratively improves the crossing angle of a given drawing. Given a different drawing of the same graph the heuristic might be able to compute a drawing with a larger crossing angle. Hence, we investigate whether the choice of the initial drawing influences the crossing angle of a drawing obtained by the RANDOM SAMPLING approach with $2 n$ iterations.

For all graph classes, except from NoRTH, it is apparent from Fig. 6 that the drawings in the set RANDOM ${ }^{\star}$ have noticeably smaller crossing angles compared to the remaining drawings. This meets our expectations, since the initial RANDOM drawings presumably has many crossings [15] and thus is likely to have many small crossing angles; compare the initial crossing angles plotted in Fig. 3 .

The plot in Fig. 6 and the characteristics in Tab. 3 in the Appendix suggest that the set $\mathrm{Fr}+\mathrm{COS}^{\star}$ contains drawings with the largest crossing angles. In 


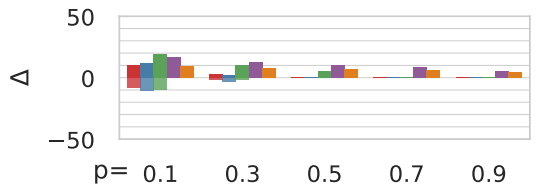

(a) STRESS $^{\star}$ vs Fr $+\operatorname{Cos}^{\star}$

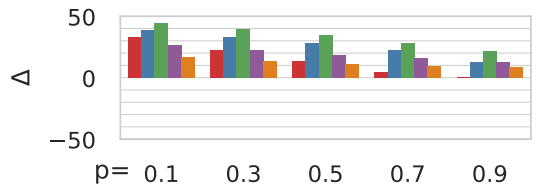

(c) RANDOM ${ }^{\star}$ vs $\mathrm{Fr}+\mathrm{COS}^{\star}$

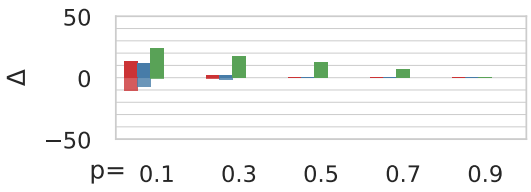

(b) CR-SMALL ${ }^{\star}$ vs $\mathrm{Fr}+\mathrm{COS}^{\star}$

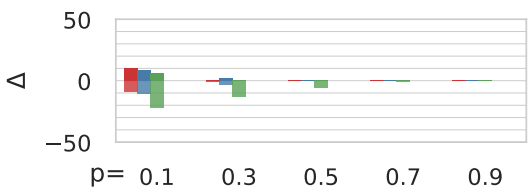

(d) Stress* vs CR-SMALL*

Fig. 9: Comparison of the crossing angle of the final drawings.

order to corroborate this claim, Fig. 8 shows crossing angles obtained by different algorithms. It shows that except for one graph, each drawing in $\mathrm{FR}+\mathrm{Cos}^{\star}$ has a larger crossing angle than the corresponding drawing in RANDOM ${ }^{\star}$. Fig $8 \mathrm{~b}$ and Fig. $8 \mathrm{c}$ suggest that $\mathrm{FR}+\mathrm{Cos}^{\star}$ overall contains more drawings with a larger crossing angle compared to STRESS ${ }^{\star}$ and CR-SMALL ${ }^{\star}$. With the help of Fig. 9 we are able to quantify the number of graphs above the diagonal and the difference of the crossing angles. Fig. 9a shows that for the graph classes 1-PLANAR and Triangulation $+\mathrm{X}$, there are each at least 90 of 100 graphs whose drawings in $\mathrm{FR}+\mathrm{COS}^{\star}$ have a crossing angle larger then the corresponding drawing in Stress $^{\star}$, i.e., Fr $+\mathrm{COS}^{\star}$ has an advantage of $4.5^{\circ}$ degrees over STRESS ${ }^{\star}$.

There are at least 50 1-PLANAR graphs such that the $\mathrm{FR}+\mathrm{COS}^{\star}$ has an advantage of $10^{\circ}$ over STRESS ${ }^{\star}$. At least 50 COMMUNITY graphs have drawings in $\mathrm{FR}+\mathrm{COS}^{\star}$ with an advantage of $5^{\circ}$ over the corresponding drawings in STRESS ${ }^{\star}$. There are 10 NORTH graphs such that $\mathrm{FR}+\mathrm{COS}^{\star}$ has an advantage of at least $5^{\circ}$ over STRESS ${ }^{\star}$. Vice versa there are 10 different NoRTH graph such that STRESS ${ }^{\star}$ has an advantage of at least $5^{\circ}$ degrees over $\mathrm{FR}+\mathrm{COS}^{\star}$. Considering subsets of size $10, \mathrm{FR}+\mathrm{COS}^{\star}$ has an advantage of $20^{\circ}$ over $\mathrm{STRESS}^{\star}$.

Recall that CR-SMALL* does neither contain drawings of the class 1-PLANAR nor of the class Triangulation $+\mathrm{X}$. The drawings of $\mathrm{FR}+\mathrm{COS}^{\star}$ has an advantage of over $7^{\circ}$ over CR-SMALL ${ }^{\star}$ on over 70 COMMUNiTY graphs. For a subset with at least 10 Community graphs, the advantage rises to almost $25^{\circ}$. The comparison on STRESS ${ }^{\star}$ and CR-SMALL ${ }^{\star}$ shows that drawings with a few crossings do not necessarily yield larger crossing angles. Overall, we conclude that the RANDOM SAMPLING approach computes the largest crossing angle when applied to the $\mathrm{FR}+\mathrm{Cos}$ drawings. This is plausible, since the crossing angles of the initial crossing angles are already good. As shown in the previous section, depending on the graph class, there is a large improvement in the crossing angle, if we start with such an initial drawing. In Appendix F we show that the 


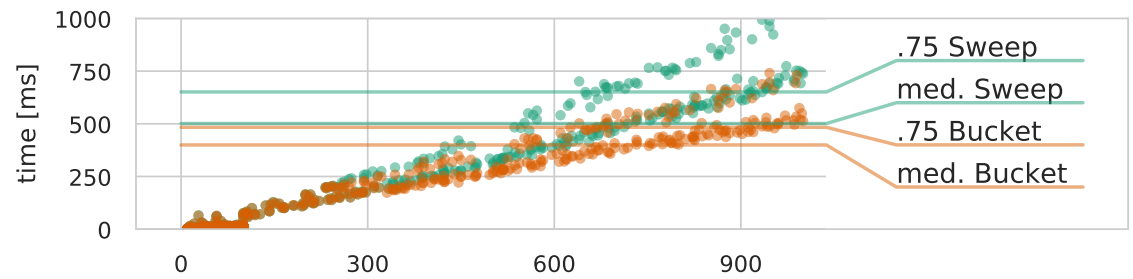

Fig. 10: Average Running time per iteration vs the number of vertices.

advantages of $\mathrm{FR}+\mathrm{CoS}^{\star}$ decreases comparably to STRESS ${ }^{\star}$ with $4 n$ iterations. However, doubling the iterations does not entirely cover the gap between the crossing angles of the initial drawings.

\subsection{Note on the Running Time}

In this section we shortly evaluate the running time of our algorithm on all our graphs. For this purpose, we applied two implementations of the RANDOM SAMPLING heuristic to the RANDOM drawings. The SweEP implementation uses a sweep-line algorithm to compute the pair of crossing edges that create the smallest crossing. BUCKET uses the algorithm described in Sec. 3.1. We employ the speed-up technique only for graphs with at least 1000 edges, we refer to these graphs as large. Fig 10 plots the running time per iteration for $n$-vertex graphs. The median and the second 3-quantile of the running time on the large graphs are highlighted. BUCKET has an average running time of $391 \mathrm{~ms}$ per iteration on the large graphs and SwEEP has an average running time of 500ms. On all graph BUCKET requires on average $328 \mathrm{~ms}$ per iteration. In Appendix G we compare the number of crossings with the number of tested edge-pairs.

\section{Conclusion}

We designed and evaluated a simple heuristic to increase the crossing angle in a straight-line drawing of a graph. On real world networks our heuristic is

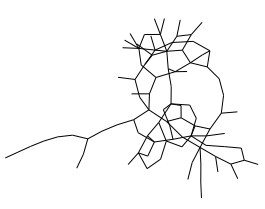

(a)

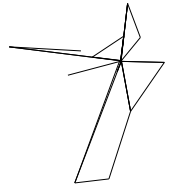

(b)

Fig. 11: (a) STRESS drawing of a Rome graph. (b) Drawing after optimizing the crossing angle. The ratio between the longest and shortest edges is large. 
able to compute larger crossing angles than on artificial networks. This can either be a limitation of our heuristic or the crossing angle of our artificial graph classes are small. We are not aware of lower and upper bounds of the crossing angle of these graphs. Thus, investigating such bounds of the 1-PLANAR and Triangulation $+\mathrm{X}$ graphs is an interesting theoretical question.

Fig. 11 shows that our heuristic does not necessarily compute readable drawings. Nevertheless, parts of the RANDOM SAMPLING heuristic are easily exchangeable. For example, the objective function can be replaced by a linear combination of number of crossing and the crossing angle. Thus, future work can be concerned with adapting the RANDOM SAMPLING approach with the aim to compute readable drawings.

\section{References}

1. Argyriou, E.N., Bekos, M.A., Symvonis, A.: The Straight-Line RAC Drawing Problem is NP-Hard. Journal of Graph Algorithms and Applications 16(2), 569-597 (2012). https://doi.org/10.7155/jgaa.00274

2. Argyriou, E.N., Bekos, M.A., Symvonis, A.: Maximizing the Total Resolution of Graphs. The Computer Journal 56(7), 887-900 (2013). https://doi.org/10.1093/comjnl/bxs088

3. Arikushi, K., Fulek, R., Keszegh, B., Morić, F., Tóth, C.D.: Graphs that admit Right Angle Crossing Drawings. Computational Geometry: Theory and Applications 45(4), 169 - 177 (2012). https://doi.org/10.1016/j.comgeo.2011.11.008

4. Bentley, J.L., Ottmann, T.A.: Algorithms for Reporting and Counting Geometric Intersections. IEEE Transactions on Computers C-28(9), 643-647 (1979)

5. Chimani, M., Gutwenger, C., Jünger, M., Klau, G.W., Klein, K., Mutzel, P.: Handbook of Graph Drawing and Visualization, chap. The Open Graph Drawing Framework (OGDF), pp. 543-569. Chapman and Hall/CRC (2013)

6. Devanny, W., Kindermann, P., Löffler, M., Rutter, I.: Graph Drawing Contest Report. In: Frati, F., Ma, K.L. (eds.) Proceedings of the 25 International Symposium on Graph Drawing and Network Visualization (GD'17). pp. 575-582. SpringerVerlag Springer International Publishing (2018)

7. Didimo, W., Eades, P., Liotta, G.: Drawing Graphs with Right Angle Crossings. Theoretical Computer Science 412(39), 5156-5166 (2011). https://doi.org/10.1016/j.tcs.2011.05.025

8. Didimo, W., Liotta, G.: The Crossing-Angle Resolution in Graph Drawing, pp. 167-184. Springer New York (2013). https://doi.org/10.1007/978-1-4614-0110$0 \_10$

9. Dujmović, V., Gudmundsson, J., Morin, P., Wolle, T.: Notes on Large Angle Crossing Graphs. In: Viglas, T., Potanin, A. (eds.) Proceedings of the 16th Symposium on Computing: The Australasian Theory (CATS'10). pp. 19-24. Australian Computer Society (2010)

10. Eades, P.: A heuristic for graph drawing. Congressus Numerantium 42, 149-160 (1984)

11. Fruchterman, T.M.J., Reingold, E.M.: Graph Drawing by Force-Directed Placement. Software: Practice and Experience 21(11), 1129-1164 (1991). https://doi.org/10.1002/spe.4380211102 
12. Gansner, E.R., Koren, Y., North, S.: Graph Drawing by Stress Majorization. In: Pach, J. (ed.) Proceedings of the 12th International Symposium on Graph Drawing and Network Visualization (GD'04). pp. 239-250. Springer-Verlag (2005). https://doi.org/10.1007/978-3-540-31843-9_25

13. Giacomo, E.D., Didimo, W., Eades, P., Hong, S.H., Liotta, G.: Bounds on the Crossing Resolution of Complete Geometric Graphs. Discrete Applied Mathematics 160(1), 132 - 139 (2012). https://doi.org/10.1016/j.dam.2011.09.016

14. Huang, W., Eades, P., Hong, S.H., Lin, C.C.: Improving Force-Directed Graph Drawings by Making Compromises Between Aesthetics. In: Hundhausen, C.D., Pietriga, E., Díaz, P., Rosson, M.B. (eds.) Proceedings of the IEEE Symposium on Visual Languages and Human-Centric Computing (VL/HCC2010). pp. 176183. IEEE Computer Society (2010). https://doi.org/10.1109/VLHCC.2010.32

15. Huang, W., Huang, M.: Exploring the Relative Importance of Crossing Number and Crossing Angle. In: Proceedings of the 3rd International Symposium on Visual Information Communication (VINCI '10). pp. 10:1-10:8. ACM (2010). https://doi.org/10.1145/1865841.1865854

16. Jonathan Klawitter and Tamara Mchedlidze and Martin Nöllenburg: Experimental Evaluation of Book Drawing Algorithms. In: Proceedings of the 25 International Symposium on Graph Drawing and Network Visualization (GD'16). pp. 224-238 (2017). https://doi.org/10.1007/978-3-319-73915-1 19

17. Lancichinetti, A., Fortunato, S., Radicchi, F.: Benchmark graphs for testing community detection algorithms. Physical Review E 78, 046110 (2008). https://doi.org/10.1103/PhysRevE.78.046110

18. Radermacher, M., Reichard, K., Rutter, I., Wagner, D.: A Geometric Heuristic for Rectilinear Crossing Minimization. In: Proceedings of the 20th Workshop on Algorithm Engineering and Experiments (ALENEX'18). pp. 129-138 (2018). https://doi.org/10.1137/1.9781611975055.12

19. Staudt, C.L., Sazonovs, A., Meyerhenke, H.: NetworKit: A tool suite for large-scale complex network analysis. Network Science 4(4), 508-530 (2016). https://doi.org/10.1017/nws.2016.20 


\section{A 1-Planar Graphs}

Fig. 12 shows the crossing angle for the geometric and topological 1-planar graphs computed with the random sampling approach. The plot already suggest that the distributions do not differ to much. Indeed, a t-test confirms that there is no significant difference between the median of the two distributions.

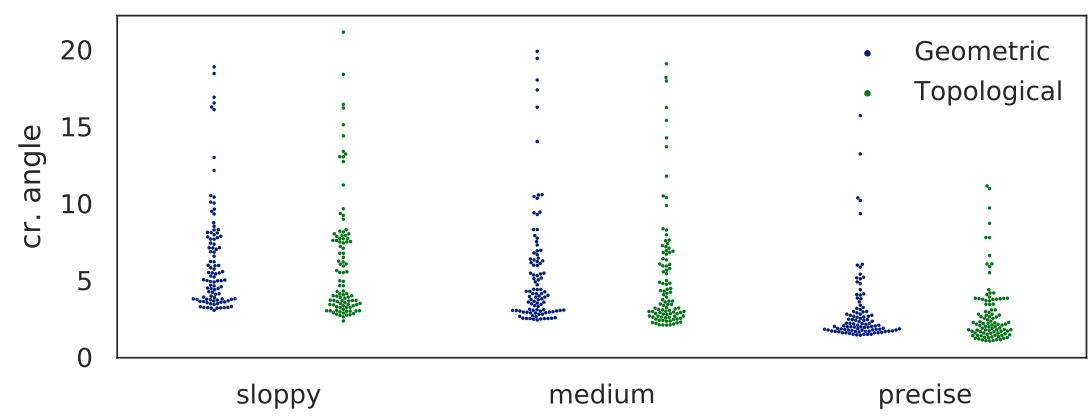

Fig. 12: Results for the two 1-planar graph classes 


\section{B Cage and Angular Force}

In this section, we evaluate the crossing angle of drawings computed by forcedirected methods applied to a random initial drawing. Stress and FMMM are implementations provided by the OGDF library. The cage drawings are obtained by the force-directed method of Fruchtermann and Reingold [11] with the additional forces $F_{\text {cage }}$ and $F_{\text {ang }}$. The drawings in $\mathrm{Fr}+\mathrm{Cos}$ use the $F_{\text {cos }}$ force instead of $F_{\text {cage }}$ and $F_{\text {ang. }}$. We use our own implementation of the of the force-directed method of Fruchtermann and Reingold.

The plot in Fig. 13 shows the final crossing angles. It indicates that the cage force produces drawings with the smallest crossing angles. This is not in accord with the claim of Argyriou et al. 2] that they obtained drawings with the largest crossing angle using their implementation of the forces $F_{\text {cage }}$ and $F_{\text {ang }}$. Our results are not necessarily comparable, since we may have used different constants to scale the forces. Moreover, we start from different initial drawings. We always start with a random drawing where Argyriou et al. use an organic layout (SmartOrganic) provided by yEd (www.yworks.com).

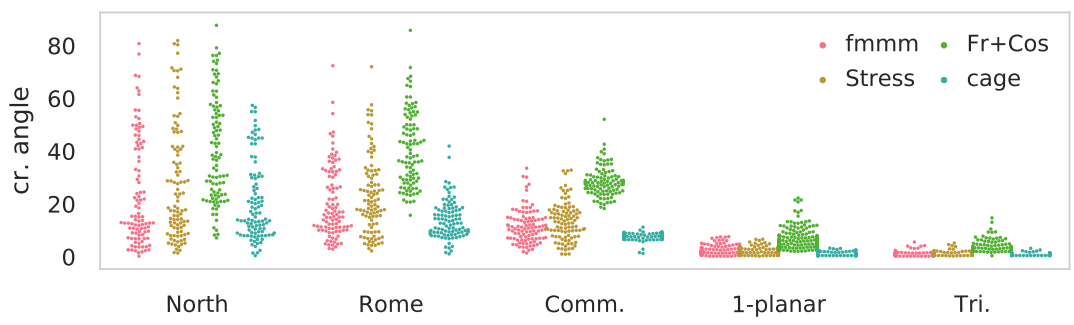

Fig. 13: Comparing Force Directed Methods

\section{Characteristics}

Tab.2 shows several characteristics of crossing angles computed with the SLOPPY, MEDIUM and PRECISE configuration of the random sampling approach. Tab. 3 shows characteristics of the crossing angle after the application of the PRECISE configuration to different initial drawings. 
Table 2: Characteristics computed by different configurations.

\begin{tabular}{|c|c|c|c|c|}
\hline g & thm & $\begin{array}{l}\text { cro } \\
\min \end{array}$ & sing & $\begin{array}{l}\text { solution } \\
\text { edian max }\end{array}$ \\
\hline Comm. & MEDIUM & 29.13 & 51.14 & 52.2381 .27 \\
\hline Comm. & Precise & 32.63 & 52.01 & 52.0778 .70 \\
\hline Comm. & SLOPPY & 28.90 & 51.12 & 51.6675 .61 \\
\hline North & MEDIUM & 20.63 & 67.12 & 63.8790 .00 \\
\hline North & Precise & 22.06 & 67.82 & 68.6990 .00 \\
\hline North & SLOPPY & 22.49 & 65.84 & 60.0090 .00 \\
\hline Rome & MEDIUM & 36.58 & 67.85 & 60.0090 .00 \\
\hline Rome & Precise & 37.97 & 66.43 & 60.0090 .00 \\
\hline Rome & SLOPPY & 32.52 & 64.86 & 59.9890 .00 \\
\hline 1-planar & MEDIUM & 4.33 & 9.02 & 7.3625 .79 \\
\hline 1-planar & Precise & 3.92 & 8.60 & 6.9726 .84 \\
\hline 1-planar & SLOPPY & 4.58 & 8.71 & 7.2322 .50 \\
\hline Tri. & MEDIUM & 5.27 & 8.94 & 7.6622 .03 \\
\hline Tri. & Precise & 4.90 & 8.55 & 7.5820 .88 \\
\hline Tri. & SLOPPY & 5.13 & 8.90 & 7.5523 .71 \\
\hline
\end{tabular}

Table 3: Characteristics

\begin{tabular}{|c|c|c|c|c|}
\hline graph & ayout & $\begin{array}{l}\text { cro } \\
\text { min }\end{array}$ & $\begin{array}{l}\text { ssing } \\
\text { mean }\end{array}$ & $\begin{array}{l}\text { solution } \\
\text { edian max }\end{array}$ \\
\hline Comm. & $\mathrm{FR}+\mathrm{Cos}^{\star}$ & 49.16 & 70.63 & 71.1088 .25 \\
\hline Comm. & RANDOM $^{\star}$ & 27.18 & 37.09 & 37.2445 .68 \\
\hline Comm. & $\mathrm{CR}-\mathrm{SMALL}^{\star}$ & 44.09 & 58.54 & 58.0384 .61 \\
\hline Comm. & STRESS $^{\star}$ & 42.89 & 65.91 & 63.7589 .09 \\
\hline North & $\mathrm{FR}+\operatorname{Cos}^{\star}$ & 23.82 & 71.29 & 78.8390 .00 \\
\hline North & RANDOM $^{\star}$ & 17.81 & 55.87 & 54.5190 .00 \\
\hline North & CR-SMALL $^{\star}$ & 18.77 & 70.55 & 87.8790 .00 \\
\hline North & StRESS $^{\star}$ & 24.46 & 70.84 & 84.6890 .00 \\
\hline Rome & $\mathrm{FR}+\mathrm{Cos}^{\star}$ & 44.52 & 77.16 & 81.2890 .00 \\
\hline Rome & RANDOM $^{\star}$ & 28.14 & 49.94 & 47.2588 .43 \\
\hline Rome & CR-SMalL ${ }^{\star}$ & 44.19 & 76.32 & 84.2890 .00 \\
\hline Rome & StRESS $^{\star}$ & 44.55 & 77.09 & 82.7090 .00 \\
\hline 1-planar & $\mathrm{FR}+\mathrm{Cos}^{\star}$ & 13.76 & 26.55 & 25.2553 .26 \\
\hline 1-planar & RANDOM $^{\star}$ & 4.55 & 6.91 & 6.0216 .67 \\
\hline 1-planar & STRESS $^{\star}$ & 9.38 & 15.81 & 13.8535 .50 \\
\hline Tri. & $\mathrm{FR}+\mathrm{Cos}^{\star}$ & 7.43 & 18.77 & 17.2436 .13 \\
\hline Tri. & RANDOM $^{\star}$ & 4.92 & 6.79 & 6.2015 .94 \\
\hline Tri. & STRESS $^{\star}$ & 6.14 & 11.95 & 10.4126 .89 \\
\hline
\end{tabular}




\section{Number of Iterations}

Fig. 14 compares the number of vertices of a graph with the number of iterations done in the given time limit. The color indicate the configuration; green is Sloppy, orange is Medium, purple is Precise. It shows that independent of the configuration, on each $n$-vertex graph at least $n$ iterations have been performed. The actual number of iterations depend on the configuration. Especially, the SLOPPY configuration is able to do at least $10 n$ iterations on most graphs. On the other hand, PRECISE moves considerably less vertices.

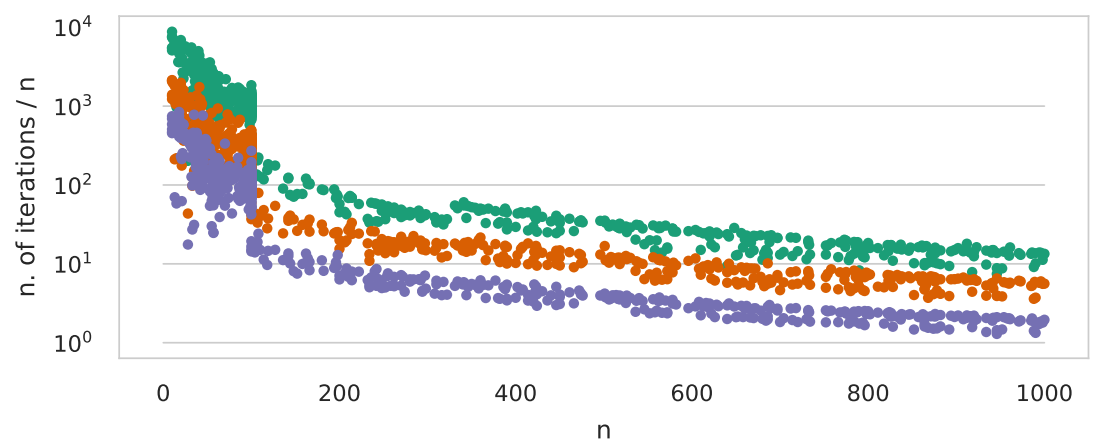

Fig. 14: Number $n$ of vertices vs. number of iterations. 


\section{E Improvement of the Crossing Angle}

Fig. 15 shows the missing data of the plot in Fig. 7, i.e., the crossing angles of the graph classes Rome and Triangulation $+\mathrm{X}$. Fig. 16 shows the corresponding advantages. The plots confirm that our approach significantly increases the crossing angles of the in the initial drawings.

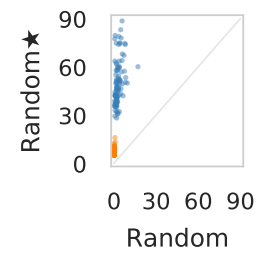

(a) RANDOM

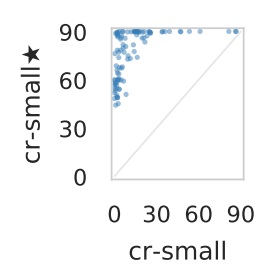

(b) CR-SMall

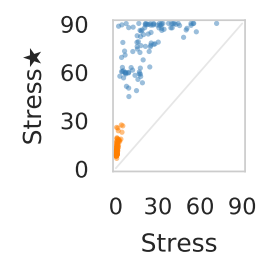

(c) Stress

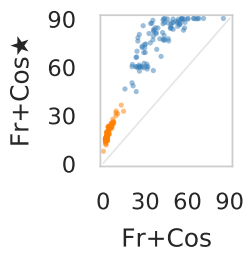

(d) $\mathrm{Fr}+\mathrm{Cos}$

Fig. 15: Initial crossing angle vs the final crossing angle. The plots show the crossing angles of the classes Rome and Triangulation $+\mathrm{X}$.

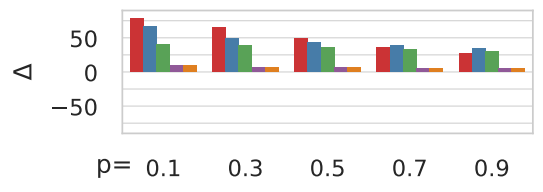

(a) RANDOM vs RANDOM*

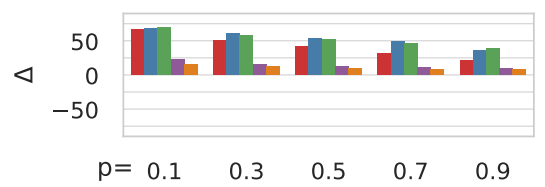

(c) Stress vs Stress*

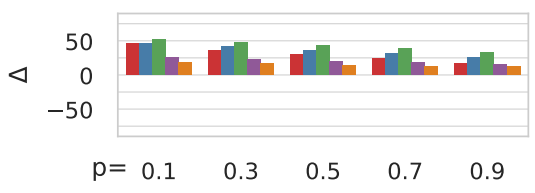

(b) $\mathrm{Fr}+\mathrm{Cos}$ vs $\mathrm{Fr}+\mathrm{Cos}^{\star}$

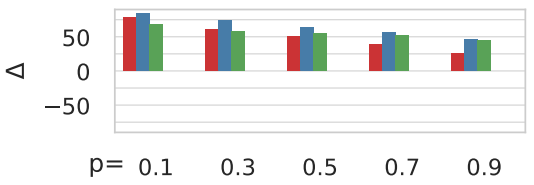

(d) CR-Small vs CR-Small ${ }^{\star}$

Fig. 16: Drawings of the initial sets vs drawings after the application of the random sampling approach. 


\section{F Increasing the Number of Iterations}

In this section we compare the following two drawings. We start with STRESS drawings and apply $4 n$ iterations with the RANDOM SAMPLING approach with the Precise configuration (STRESS ${ }^{\star \star}$ ). Second, we apply $2 n$ iterations to the $\mathrm{FR}+$ Cos drawings. The plots in Fig. 17 show that drawings of graphs in NORTH and Rome have indeed a slightly larger crossing angle when they are obtained from STREss. For the remaining graph classes the drawings obtained Fr + Cos still have an advantage over STRESS.

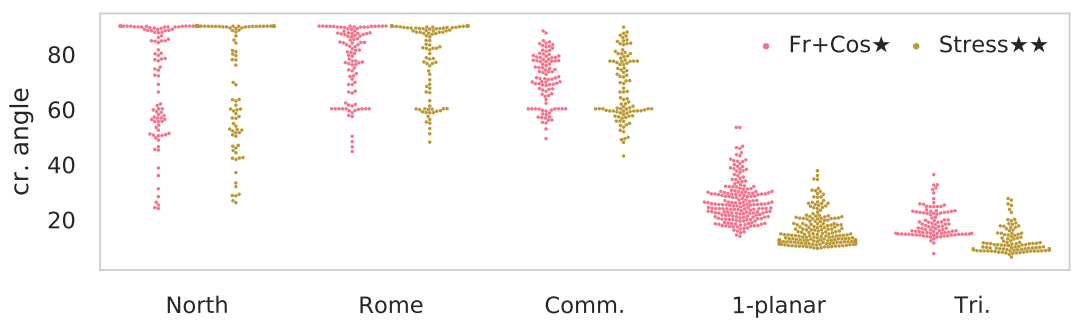

(a)

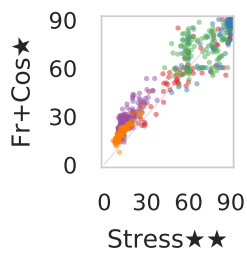

(b)

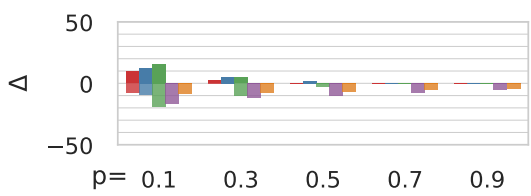

(c) $\mathrm{Fr}+\mathrm{Cos}^{\star}$ vs STRESS ${ }^{\star \star}$

Fig. 17: Comparison 


\section{G Number of Crossings vs Number of Operations}

In this section, we further evaluate our speed-up heuristic introduced in Section 3.1. We compare the number of crossings $C$ in a drawing with the number $P$ of edge-pairs that the speed-up heuristics tests for intersections. Ideally, $P$ is significantly smaller than $C$.

One data point in Fig. 18 corresponds to a single graph. We applied the SLOPPY heuristic to the random drawings of all our benchmark graphs. We counted in each iteration the number of crossings of the current drawing and the number of tested pairs. The values in the figure correspond to the average of these values over $2 n$ iterations. Note that the plot uses a double log-scale.

We observe that for small instances, the heuristics tests more edge-pairs than the drawing has crossings. With an increasing number of crossings, the heuristic indeed tests less edge-pairs than crossings.

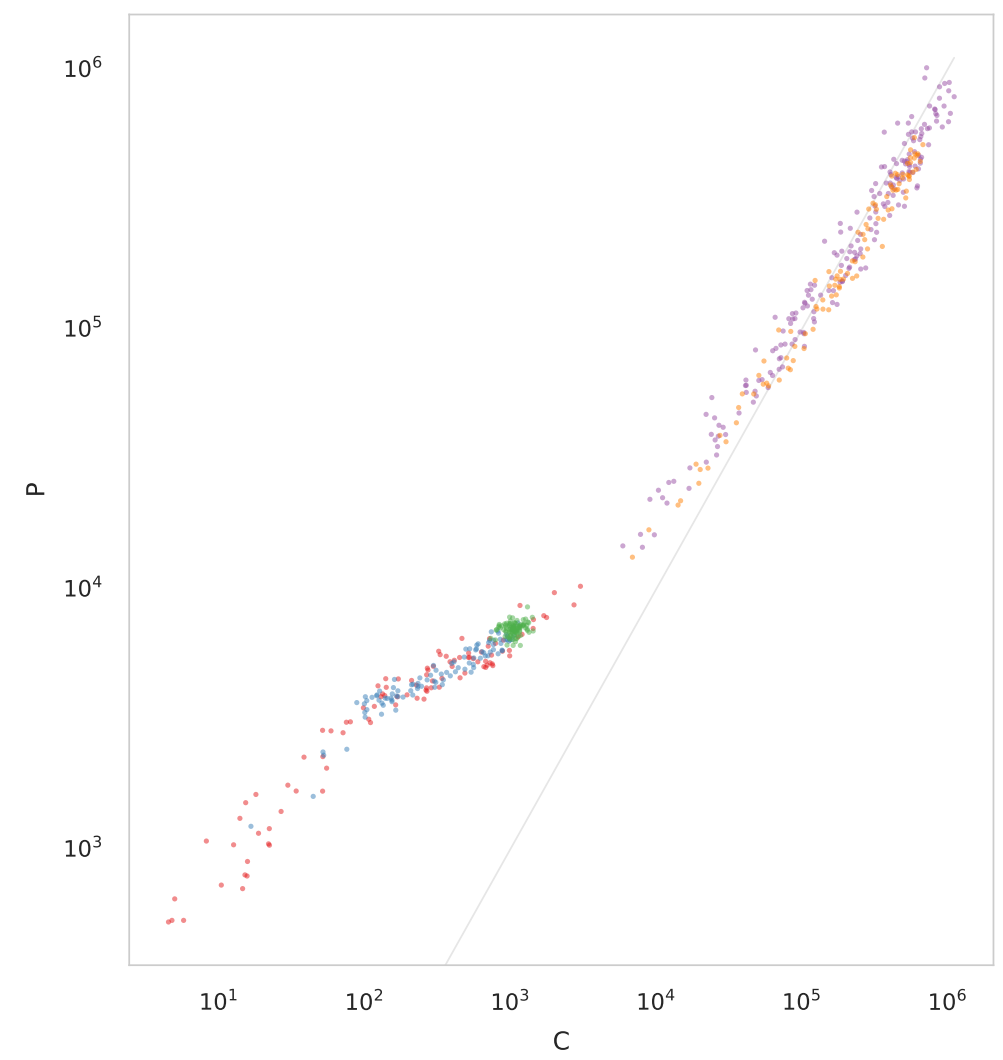

Fig. 18: Number $C$ of crossings vs number $P$ of tested edge-pairs. 